\title{
Noninvasive ventilation for acute exacerbations of chronic obstructive pulmonary disease in the emergency department: early predictors of in-hospital outcome
}

\author{
R. Ferrari, F. Giostra, D. Agostinelli, G. Monti, D. P. Pomata, M. Cavazza
}

Unità Operativa di Medicina d'Urgenza e Pronto Soccorso. Policlinico Sant'Orsola - Malpighi. Dipartimento Emergenza / Urgenza, Chirurgia Generale e dei Trapianti. Azienda Ospedaliero Universitaria di Bologna. Università degli Studi di Bologna, Alma Mater Studiorum

\section{Introduction}

The applications of early non-invasive ventilation (NIV) are nowadays expanding in the Emergency Department (ED) for the treatment of different types and etiologies of acute respiratory failure (ARF)

Emergency physicians (EP) often face the challenge of predicting short-term outcomes for patients with ARF in the ED

\section{Aim}

To critically analyze and describe the impact of NIV and the peculiar characteristics connected to its use in the ED, with specific attention to ARF presentation due to acute exacerbation of chronic obstructive pulmonary disease (AECOPD)

To identify early and objective clinical parameters and laboratory biomarkers for severity assessment and predicting the outcomes in AECOPD requiring NIV

\section{Methods}

Three-months observational prospective clinical single-centre study in real life practice of the acute setting of the ED of a university teaching hospital, including every consecutive non-selected patient emergently admitted for ARF due to AECOPD, and treated according to EP's early clinical indication to first-line NIV instead of conventional oxygen therapy or IMV, referring to an institutional protocol

We prospectively analyzed and then retrospectively abstracted medical, clinical and gas-analytic data recorded at presentation and 30 to 360 minutes after starting the treatment, and the outcome at the end of hospitalization

Treatment failure was defined as hospital mortality and / or need for tracheal intubation (TI) and invasive mechanical ventilation (IMV) at any time

\section{Results}

124 patients (media 1.38/day). Failure (23 cases, 18.5\%) and success (101; 81.5\%) patients were different in: neurologic status score (Kelly-Matthay scale), urea, creatinin, AST, ALT, CPK, CPK-MB, troponin T, LDH, PCR, pH, and arterial blood gas analysis parameters after 1-2 hours of NIV (PaO2, pH, PaCO2, HCO3-, SaO2, PaO2/FIO2).

\section{Conclusions}

NIV in the ED is a cost effective intervention, well tolerated, easy to use, low costly to administer, has few side effects, few complications and reduced need for sedation, even outside intensive care units for the treatment of ARF caused by AECOPD. In our ED the global safety and efficacy of NIV in avoiding TI and improving the outcome of carefully selected and controlled patients admitted for ARF is confirmed and is similar to what has been documented in previous RCTs

We showed the possibility to identify in the ED early predictors of outcome: some clinical parameters, biomarkers and arterial blood gas analysis data to recognize severe conditions and the response to treatment

An appropriate patient selection and a properly timed intervention are required for the optimal administration of safe and effective early NIV in haemodynamically stable patients, not yet meeting criteria for IMV, who can be closely monitored in an environment where TI is promptly available, with a medical and nursing motivated team with an extensive training and experience in NIV

Unnecessary delaying TI remains the major hazard of NIV in ARF. A main unresolved question is about selection criteria and early choices for patients with ARF having preset therapeutic and prognostic limits and acutely reversible processes for which NIV should be considered as a "ceiling" treatment 